\title{
ARTICLE
}

\section{A dose-finding study of oxytocin using neurophysiological measures of social processing}

\author{
Jonathan K. Wynn $\mathbb{D}^{1,2}$, Michael F. Green ${ }^{1,2}$, Gerhard Hellemann $\mathbb{D}^{2}$, Eric A. Reavis ${ }^{1,2}$ and Stephen R. Marder $\mathbb{D}^{1,2}$
}

Recent interest has focused on oxytocin (OT), a neurotransmitter that promotes social processing, to improve social functioning in people with schizophrenia. However, little information is available regarding the doses of OT that are effective for influencing social processing in the brain (i.e., target engagement). In this study, we conducted a double-blind, placebo-controlled, cross-over dose ranging study of OT. In total 47 patients with schizophrenia were randomly assigned to one of eight doses of OT (8, 12, 24, 36, 48, 60,72 , or $84 \mathrm{IU}$ ). Patients completed two social processing tasks: one electroencephalography (EEG) task, a biological motion Musuppression task (i.e., identifying the gender, emotion, or direction of walking of point-light animations of human movement); and one pupillometry task, pupil dilation in response to viewing affective faces. Participants completed these tasks twice, one week apart, and were randomly administered drug or placebo intranasally $30 \mathrm{~min}$ prior to each session. Mu-suppression, i.e., suppression of oscillations in the $8-12 \mathrm{~Hz}$ range over central electrodes in response to social stimuli, was significantly enhanced at doses of 36 and $48 \mathrm{IU}$ in comparison to placebo, but not at other doses. Significant pupil dilation was observed in response to faces vs. non-face stimuli, though there were no drug effects at any dose. Results suggest that OT affects central measures of social information processing in patients with schizophrenia and is optimal at a mid-range dose (36-48 IU). These results provide dosing guidance for future studies of OT to be used to enhance social processing in people with schizophrenia.

Neuropsychopharmacology (2019) 44:289-294; https://doi.org/10.1038/s41386-018-0165-y

\section{INTRODUCTION}

Oxytocin (OT), a neuropeptide and neurotransmitter, regulates certain social behaviors in mammals including social bonding [1-3]. Recent research has evaluated whether intra-nasally administered OT can improve social processing in disorders that are characterized by impairments in this area, such as autism and schizophrenia [4, 5]. However, progress in this area has been limited by a lack of knowledge regarding the most effective dose of OT for affecting social processing. For example, in a recent summary of 14 clinical trials of OT in schizophrenia, substantial inconsistencies in the doses of OT were noted, with doses ranging from 10 to $80 \mathrm{IU}$ [4]. It is unclear if all of these doses are in a clinically effective range. To reach meaningful conclusions about OT's therapeutic value, it is important to understand the effects of different doses on brain targets that affect the processing of social information. Such information about OT's dosing and effects on brain processing is consistent with an experimental medicine approach and the current clinical trial requirements at the National Institute of Mental Health (NIMH). Specifically, NIMHfunded clinical trials require that studies demonstrate target engagement, broadly defined as evidence that an effective dose can be demonstrated on a proposed mechanism [6, 7].

Neurophysiological methods from affective neuroscience may be useful for demonstrating target engagement of OT. For example, electroencephalography (EEG) has been used to examine the effects of OT on Mu suppression during a biological motion task. Mu suppression is a reduction in activity over central electrode sites in the $8-13 \mathrm{~Hz}$ range in response to social vs. nonsocial stimuli [8], and is thought to reflect activity of the mirror neuron system [9]. However, while Mu suppression does appear to be an index of social processing the connection to the mirror neuron system is more debatable [10]. When given $24 \mathrm{IU}$ of OT, healthy participants exhibited significantly enhanced Mu suppression to biological motion stimuli compared to placebo [11], indicating that OT enhanced processing of social stimuli. Pupillometry has been another method to examine the effects of OT on social stimuli. Typically, greater pupil dilation occurs when processing facial emotional content $[12,13]$ vs. non-face content. When administered 24 IU of OT, healthy participants exhibited enhanced pupil dilation to facial stimuli compared to those on placebo [14], again demonstrating that OT enhances processing of social stimuli.

In the current study, we conducted a dose finding study of OT in patients with schizophrenia, utilizing EEG and pupillometry, to demonstrate target engagement. Patients received either placebo or one of eight different doses of OT in separate test sessions one week apart; order of test session (e.g., OT then placebo) and dose were both randomized. For target engagement, we measured $\mathrm{Mu}$ suppression during a biological motion task and pupillary responses during a facial affect identification task. We hypothesized that patients would show the expected pattern of results on the two tasks during the placebo condition (i.e., Mu suppression to social vs. non-social stimuli, pupil dilation to face vs. non-face stimuli), demonstrating task validity. We also hypothesized that

\footnotetext{
${ }^{1}$ Mental Illness Research, Education and Clinical Center, Veterans Affairs Greater Los Angeles Healthcare System, Los Angeles, CA, USA and ${ }^{2}$ Semel Institute for Neuroscience and Human Behavior, University of California, Los Angeles, Los Angeles, CA, USA
}

Correspondence: Jonathan K. Wynn (jkwynn@ucla.edu)

Received: 7 March 2018 Revised: 13 July 2018 Accepted: 19 July 2018 Published online: 28 July 2018 
there would be an effect of dose on our target engagement measures such that we could identify the most effective dose of OT for enhancing the processing of social information.

\section{MATERIALS AND METHODS}

\section{Participants}

Fifty-one stable outpatients with schizophrenia were recruited from the VA Greater Los Angeles Healthcare System (VAGLAHS) and outpatient clinics in the greater Los Angeles area. Eligibility criteria include: (a) DSM-5 diagnosis of schizophrenia based on clinical interview and review of medical records, (b) age 18-65 years, (c) understand spoken English sufficiently to comprehend testing procedures, (d) no clinically significant neurological disease determined by medical history (e.g., epilepsy), (e) no history of serious head injury (i.e., loss of consciousness $>1 \mathrm{~h}$, no neuropsychological sequelae, no cognitive rehabilitation post head injury), ( $f$ ) no sedatives or benzodiazepines within $12 \mathrm{~h}$ of testing, and (g) corrected vision of at least 20/30. Additional eligibility criteria included $\geq 3$ months since any psychiatric hospitalization; $\geq 6$ months since any behaviors suggesting any potential danger to self or others; currently prescribed an antipsychotic medication with dose not varying by more than $50 \%$ over the 3 months prior to study participation; no acute medical problems; and chronic medical conditions are consistently treated and stable for $\geq 3$ months prior to study participation. Four participants did not complete testing (see CONSORT chart in Fig. 1), thus the final analyzed sample size is 47 . In total 41 participants were on second generation antipsychotic medications, 4 on mixed first and second-generation medications, and medication information was not available for 2 participants.

All participants had the capacity to give informed consent and provided written informed consent in accordance with procedures approved by the Institutional Review Board at VAGLAHS and UCLA.

\section{Clinical symptom assessments}

Brief Psychiatric Rating Scale (BPRS): The UCLA expanded 24-item version of the BPRS [15] was the principle measure for assessing positive symptoms. We analyzed the total BPRS score, as well as the positive factor component [16]. All interviewers were trained at the Treatment Unit of the VISN 22 Mental Illness Research, Education and Clinical Center (MIRECC) to a minimum kappa of

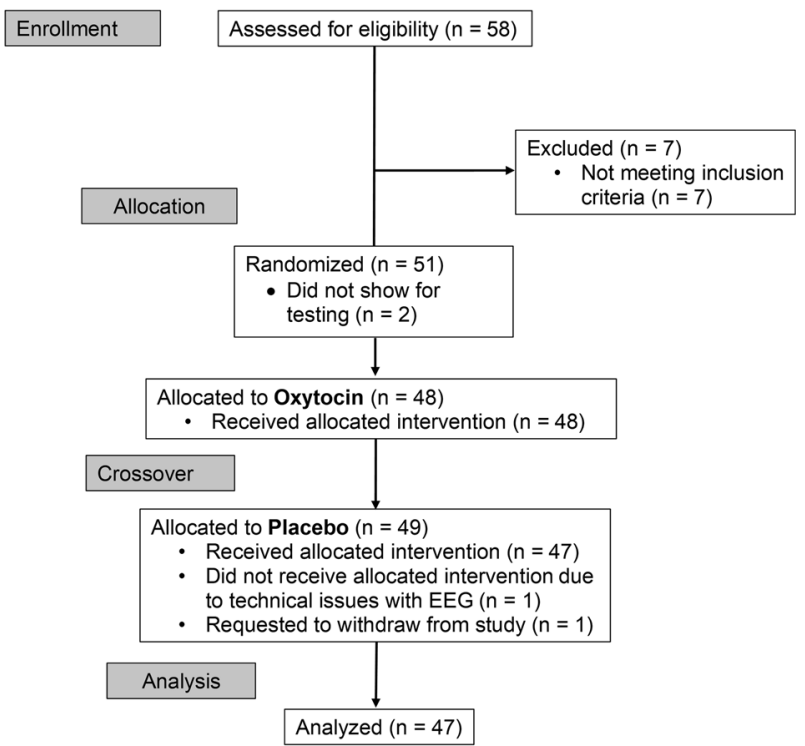

Fig. 1 CONSORT flow chart for enrollment, allocation, and analysis of data
0.75 for key psychotic and mood items. All participants received a diagnostic interview with the Structured Clinical Interview for DSM-V (SCID-I) [17].

Pharmacological treatment

OT nasal spray was compounded by Inland Compounding Pharmacy (Loma Linda, CA). A placebo nasal spray was prepared that was otherwise identical to the active treatment. Nasal sprays were prepared in $3 \mathrm{ml}$ single use vials, calibrated to dispense 0.1 $\mathrm{ml}$ per puff. Eight doses of OT were used: 8, 12, 24, 36, 48, 60, 72, or 84 IU. Six participants were assigned to each OT/placebo dose. Intranasal OT is believed to increase both central and peripheral levels in humans, with a peak response $\sim 40-50 \mathrm{~min}$ after administration [18-20]. For both OT and placebo, administration of spray was done $30 \mathrm{~min}$ prior to testing.

\section{Study design}

Informed consent, demographic data, and medical history were obtained for each participant at an initial screening visit. A brief physical examination was also performed. The design was a randomized, double-blind, placebo-controlled cross-over study with participants receiving either OT or placebo on separate testing days one week apart. Participants were randomly assigned to one of the eight doses, and administration of drug and placebo was also conducted in random order. Drug condition was concealed to all staff during the course of the trial, including during data collection and initial EEG and pupillometry processing.

Participants received EEG and pupillometry in the same sequence at both visits. Thirty minutes prior to beginning assessments, the study coordinator monitored administration of the nasal spray to each participant. After inhalation, participants were set up for the EEG assessment. Thirty minutes after inhalation, participants then received the biological motion $\mathrm{Mu}$ suppression task $(15 \mathrm{~min})$. After completion of the task, participants removed the EEG cap and moved to the facial affect pupillometry task (20 min).

All study activities were conducted at the Translational Research Center for Neuroscience at UCLA. This trial was registered on ClinicalTrials.gov with the title "Target Engagement for Oxytocin: Dose Ranging Study" and identifier: NCT02498236. Recruitment occurred between September, 2015 and July, 2017.

Biological motion Mu suppression: acquisition and analysis A biological motion task was used to assess Mu suppression. We used the same task as a previous study of OT in healthy controls [11]. Participants sat in an electrically isolated chamber. The stimuli consisted of $5 \mathrm{~s}$ video clips of point-light walkers simultaneously depicting three different dimensions, each manipulated independently: expression (happy or sad), gender (male or female), or intention (walking towards or away from the viewer). Thus, an example of a point-light walker could consist of a happy male figure walking away from the viewer, or a sad woman walking towards the viewer, etc. As a control condition, a circle constructed of point lights was used; the circle moved either to the left or to the right of the screen starting from central fixation.

Stimuli were presented in four separate, randomized blocks, with each block requiring the participant to identify only 1 of the 4 dimensions: emotion, gender, intention, or circle movement. Thus, for biological motion point light walkers the other dimensions were simultaneously present, but participants were instructed to only process one of those dimensions (e.g., emotion) and ignore the other dimensions. In each block, 26 stimuli were presented. Within a given dimension, twenty stimuli of one type and six stimuli of the other were presented (e.g., 20 male and 6 female figures, or 20 happy and 6 sad figures). At the beginning of each block participants were instructed to identify and mentally keep track of the number of rare-occurrence stimuli and report at the end of the block how many times they saw that stimulus. 
Details on EEG acquisition and processing can be found in Supplemental Methods. Mu suppression ratios were calculated over three central $(C 3, C z, C 4)$ electrodes. Each biological motion condition (emotion, gender, intention) was compared to the circle condition. Mu suppression was calculated as $\log _{10}(\mathrm{Mu}$ power to biological motion condition/Mu power to circle). Log transformation was used because ratio data are non-normally distributed, and to control for variability in absolute $\mathrm{Mu}$ power due to individual differences. A log ratio less than zero indicates $\mathrm{Mu}$ suppression, a log ratio equal to zero indicates no change, and a log ratio greater than zero indicates mu enhancement.

Facial affect pupillometry: acquisition and analysis

In this facial affect identification task 16 happy, 16 afraid, and 16 neutral pictures of Caucasian and Asian people were selected from the Matsumoto and Ekman Japanese and Caucasian Facial Expressions of Emotion stimulus set [21]. An equal number of males and females were portrayed in each condition. As a nonface control condition, 16 "scrambled" images were created from the original files using custom-written scripts in Matlab (Mathworks, Natick, MA). This technique maintained the physical properties of the image (e.g., luminance, contrast, etc.) but rendered any facial feature unrecognizable

For each trial, a screen with the word "Ready?" was presented until the participant verbally indicated readiness. Following this screen, a $3 \mathrm{~s}$ fixation cross was presented followed by a $5 \mathrm{~s}$ presentation of one stimulus from one of the four conditions (happy, afraid, neutral, or scrambled). After the stimulus presentation, a screen was presented asking the participant which of the four stimulus conditions they saw. Eight stimuli were presented for each condition in a randomized order, each repeated twice, for a total of 64 stimuli presented throughout the task.

Further details on pupillometry acquisition and processing can be seen in Supplementary Methods. Pupillometry data were continuously acquired from the right eye, sampled at $220 \mathrm{~Hz}$ using a ViewPoint head-fixed infrared eye tracker (Arrington Research, Scottsdale, AZ). Pupillometry data were processed in MATLAB (MathWorks, Natick, MA) using custom-written scripts. A onesecond period prior to the onset of the face/scrambled stimulus was extracted and processed to estimate baseline pupil size for that stimulus. Baseline was defined as the median pupil width during the one-second window, after excluding any timepoints containing eyeblinks, saccades, or other artifacts identified by the pupilometer. Pupil sizes during each trial were baseline-corrected by dividing them by baseline median width. Resulting pupil size timecourses were averaged within-subject for each type of stimulus (happy, afraid, neutral, scrambled). The primary dependent variable was computed by taking the average of the change in pupil size between 1 and $3 \mathrm{~s}$. This time window was chosen to exclude the initial pupil contraction due to the light reflex from the onset of the stimulus.

\section{Statistical analysis}

To confirm that the mu suppression and pupillometry tasks provided valid data (i.e., results were of the expected pattern), we conducted preliminary analyses using only placebo data from the participants. For the mu suppression task, we first conducted three, one-sample t-tests comparing mu suppression values for each of the three experimental conditions to zero to confirm that significant suppression was observed. Next, we conducted a repeated-measures ANOVA with condition (gender, intention, emotion) as the within-subjects factor to determine if suppression differed by identification task. For the pupillometry task, we first conducted three paired t-tests comparing each of the three face conditions (fearful, happy, neutral) to the control condition (scrambled) to determine if significantly greater pupil dilation was seen to faces compared to non-faces. Next, to examine whether pupil dilation differed across the three face conditions, we conducted a repeated-measures ANOVA with condition (fearful, happy, neutral) as the within-subjects factor.

The analysis of the dose-response function of OT on $\mathrm{Mu}$ suppression and pupil dilation was done in two steps. For each of these analyses we created a placebo response group using the placebo results across all 47 participants. In the first step, we checked to see if any of the drug dosages deviated from placebo. Our intent was to obtain data on a large range of OT doses, as the prior literature was not helpful in determining optimal dose. Consequently, we had relatively few subjects at each dose. This approach limited our power to detect differences at each specific dose, thus we decided to combine results for the placebo condition and analyze data with a GLMM to increase power to detect specific doses where OT had an effect. As there was no a priori mathematical model of an expected dose-response function, we initially created the empirical dose-response curve, and used a non-structured mixed model to estimate the confidence band of this curve. The mixed model analysis fully accounts for the repeated measure structure and takes into account the autocorrelation between the measurement in the placebo condition and the measurement in the active condition. It should be noted that degrees of freedom are estimated in this analysis using the Satterthwaite Degrees of Freedom Approximation, and thus will vary from test to test. For Mu suppression, we included condition (emotion, intention, gender) as a withinsubjects factor and dose as a between-subjects factor. We assessed for main effects of dose, condition, as well as the interaction between the two. In the second step, we then performed post-hoc analyses for Mu suppression at each dose, collapsed across conditions, comparing to placebo. For post hoc analyses, we present the estimated marginal means and standard errors in parentheses. As these are post-hoc tests following a significant omnibus result we do not correct for multiple comparisons (Fishers LSD principle). For pupillometry, we were interested in the effects of OT on processing of emotion stimuli. We first calculated a difference score between pupil sizes for the happy and fearful conditions minus the scrambled condition. We included the average of the two difference scores in the mixed model analysis, with dose as a between-subjects factor. We then performed post-hoc analyses at each dose comparing to placebo.

\section{RESULTS}

Demographics and symptom ratings appear in Table 1. Participants were predominantly male, in their low $40 \mathrm{~s}$, and exhibited mild positive and negative symptoms at the time of testing.

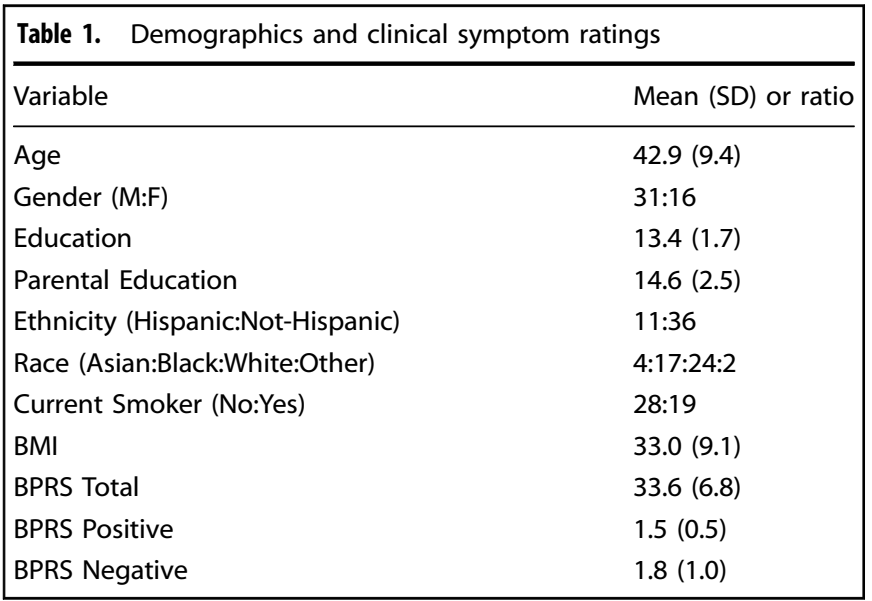




\section{Mu suppression}

To assess the validity of the task, we examined the data for the placebo condition to determine whether the three biological motion conditions exhibited Mu suppression. As can be seen in Fig. 2, all three conditions resulted in mu suppression (i.e., logtransformed ratio values comparing the control condition were negative). One-sample $t$-tests revealed that all three conditions resulted in significant mu suppression, all $t^{\prime} \mathrm{s}(\mathrm{df}=46)<-2.96, p^{\prime} \mathrm{s}$ $<0.005$. Next, we ran a repeated-measures ANOVA to examine differences in mu-suppression among the three conditions. Results revealed a significant main effect of condition, $F_{2,92}=$ $3.26, p<0.005$. Follow-up contrasts revealed that this significant main effect was due to significantly less suppression in the gender condition compared to the emotion $(p<0.03)$ condition. There was no significant difference in suppression between the emotion and intention conditions or between the intention and gender conditions.

Next, we examined for OT effects on condition. Mu suppression responses for each individual and for each condition can be seen plotted as a dot and line plot in Fig. 3. Responses are plotted at each dose and at placebo ("dose 0"), for the emotion (black circle), intention (light gray square), and gender (gray triangle) conditions. A reference line was plotted along the $y$-axis showing the mean and its $95 \%$ confidence interval averaged across all participants and all conditions for the placebo condition. Mean lines are plotted for each dose and condition separately (same color scheme described above).

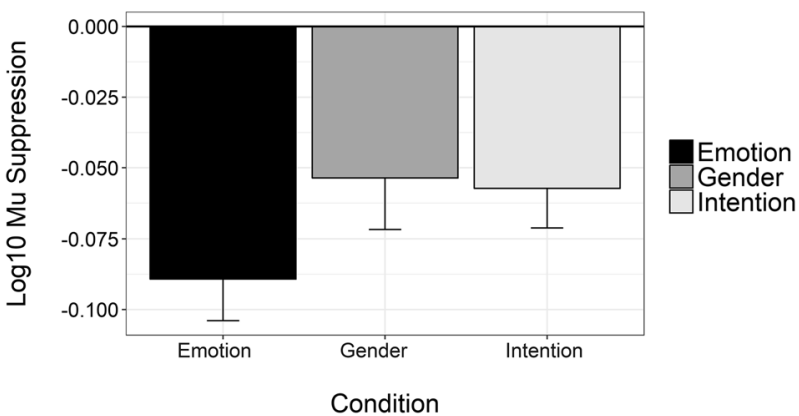

Fig. 2 Mean $\log _{10}$ Mu suppression for placebo administration only. Error bars represent \pm 1 standard error. Mean values for the emotion identification (black), gender identification (gray), and intention identification condition (light gray) are all significantly different from zero, indicating significant mu suppression occurred

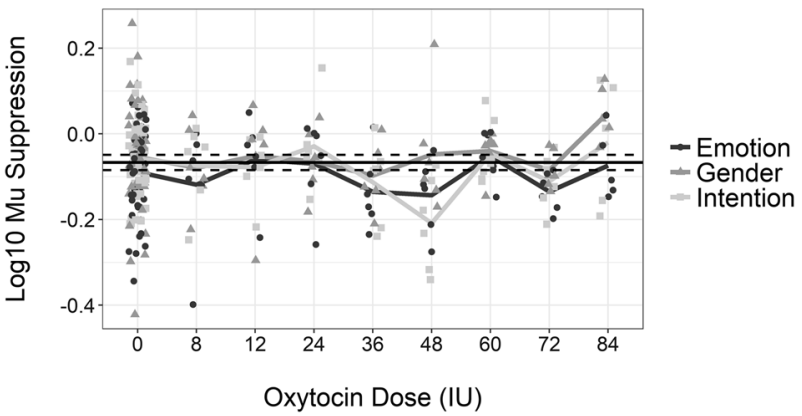

Fig. $3 \log _{10} \mathrm{Mu}$ suppression values for each observation for emotion identification (black circle), gender identification (gray triangle), and intention identification (light gray square) are shown for placebo $(I U=0)$ and the eight OT IU doses. Mean values for each dose are displayed as overlaid lines following the same color scheme. A reference line for the mean of the placebo condition (horizontal black line), along with lines for the 95\% confidence interval (dashed black lines above and below mean), is shown
Results of the mixed model analysis yielded significant effects of condition $\left(F_{2,207}=4.68, p=0.01\right)$ and dose $\left(F_{8,247}=2.69, p=0.01\right)$, but no significant interaction between the two $\left(\mathrm{F}_{16,207}=0.94, p=\right.$ 0.53 ). Post hoc analyses of the condition effect showed that mu suppression during gender identification, -0.05 (0.01), was significantly smaller compared to emotion identification, -0.10 (0.01), $p<0.01$, and intention identification, $-0.08(0.01), p=0.05$. The difference between the emotion and intention conditions was not significantly different $(p=0.32)$. Regarding dose effects, analyses showed that mu suppression at doses of $36 \mathrm{IU},-0.14$ (0.02), and $48 \mathrm{IU},-0.13(0.02)$ was significantly greater compared to the placebo condition, $-0.07(0.01), p^{\prime} s=0.01$ and 0.02 , respectively. While it appears that there could be a significant effect at $72 \mathrm{IU}$, given the similar pattern as at 36 and $48 \mathrm{IU}$, the result did not reach significance given that there was more heterogeneity in the difference between placebo and OT than at the lower doses. The significant effects can be seen in a supplementary bar chart, showing differences between OT and placebo only for those in the 36 and 48 IU doses, collapsed across identification condition (Supplementary Figure 1).

\section{Pupillometry}

To assess the validity of the task, we examined the effects of condition on pupil dilation only in the placebo condition (Fig. 4). One-sample $t$-tests (comparing against a ratio of 1 , i.e., baseline) revealed that the three face conditions resulted in significant pupil dilation, all $t^{\prime} s(\mathrm{df}=46)>2.93, p^{\prime} s<0.005$; pupil dilation to scrambled faces was not significant $(p>0.45)$. Next, we ran a repeated-measures ANOVA to determine if there were differences in pupil dilation among the four conditions. There was a significant main effect of condition $F_{3,138}=6.81, p<0.001$. Follow-up contrasts revealed that dilation was significantly smaller to the scrambled face compared to fearful and neutral ( $p^{\prime} s<0.06$ ) but not happy faces $(p=0.21)$. There were no other significant differences between the conditions. With regards to the analysis for OT effects, results of the mixed model analysis did not reveal a significant dose effect, $\mathrm{F}_{8,61}=1.06, p=0.40$. We therefore did not perform any post hoc analyses. The estimated marginal means, standard deviations, standard errors, and confidence intervals for the pupillometry data are in Supplementary table 1.

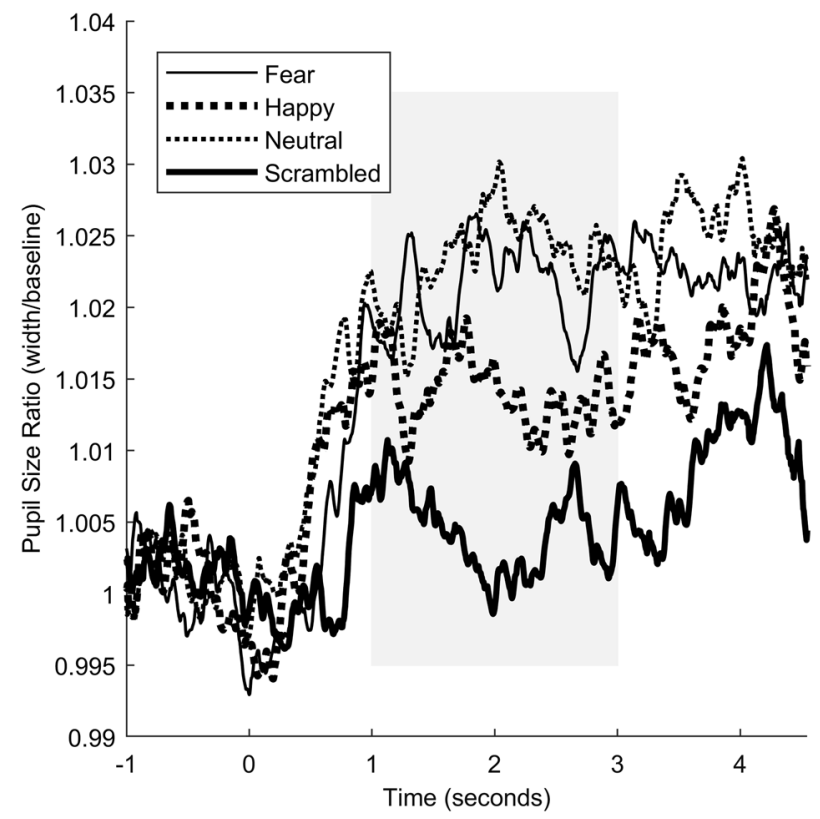

Fig. 4 Pupil responses to the facial affect identification task. Tracings are shown for responses to fearful (thin solid line), happy (large dashed line), neutral (small dashed line), and scrambled (thick solid line) faces 


\section{DISCUSSION}

In the current study we used measures of brain target engagement to identify doses of intra-nasally administered OT that had effects on social information processing. Oxytocin administered prior to testing enhanced mu suppression to biological motion stimuli at 36 and $48 \mathrm{IU}$ doses compared to placebo. We did not identify any significant OT dose ranges on pupillometry responses to affective faces compared to placebo. This is the first study to our knowledge to demonstrate target engagement and determine dosing of OT to affect social processing in people with schizophrenia.

In reviewing prior clinical trials, doses in the range of 10-80 IU have been administered, and frequently the studies use doses of 24 or $40 \mathrm{IU}$. In a dose dependency study of OT in healthy humans, using $\mathrm{fMRl}$ during an emotional face recognition task, OT significantly reduced activation in the amygdala to fearful faces at a dose of 24 IU 45-70 min after intranasal administration [22]. In the current study, the doses that produced significant enhancement of Mu suppression to social stimuli, 36 and 48 IU OT, are comparable to commonly used doses in previous studies with schizophrenia, but slightly higher than the 24 IU dose in healthy humans. Our results are consistent with those of [23] which showed significant effects of OT at $48 \mathrm{IU}$ in people with schizophrenia and at a lower dose of $24 \mathrm{IU}$ in healthy controls, though this effect was moderated by gender with significant effects at 48 in male patients only.

While we found significant OT effects for the Mu suppression task, we did not find significant effects for the facial affect pupillometry task. One potential explanation for a lack of an effect may be the well-known face processing deficits in people with schizophrenia (e.g., [24]). These deficits may be too pervasive to be affected by a one-time dose of $\mathrm{OT}$, and may require more intensive intervention, such as social cognitive skills training, in order to see improvements [25]. The lack of effects may also be due to the use of faces (Caucasian and Asian) that were not similar to a large percentage of our sample. Differential patterns of pupillary responses to other- vs. own-race faces have been documented (e.g., [26]). Future studies of OT effects on pupillary responses to faces could consider using own- vs. other-race faces. One final possible explanation is that mu suppression, assessed with EEG, is a more direct neural measure than pupillary response. Biological motion involves the superior temporal sulcus [27] and mirror neuron networks in the premotor cortex [28]. As we measured EEG on scalp regions directly over the premotor cortex, the mu suppression paradigm in the current study may have been more sensitive to OT effects.

We initially evaluated the dose-response curve as a sigmoidal response, with increased doses yielding increased response with diminishing returns. However, the empirical dose-response curve was unimodal, and could not be modeled in the typical frameworks used for dose-response curves. Instead, we attempted to fit a polynomial linear regression that has been used previously for U-shaped dose-response curves within the mixed model framework [29]. However, due to the sparseness of the data these exploratory models did not converge.

This study had a few limitations. First, all patients were receiving antipsychotic medication at the time of testing. However, this medication status is similar to the vast majority of OT studies done with people with schizophrenia. Further, the patients were in the same status of medication for the test and placebo conditions, making it unlikely that medication influenced the OT-placebo differences. Second, we had a relatively small sample size to test at each OT dose. This is particularly problematic when studying phenomena affected by factors with high variability (e.g., heterogeneity of illness, heterogeneity of drug absorption via intranasal administration). However, our use of a within-subjects design was chosen to increase power to detect significant effects with small sample sizes. While we did find significant effects of OT at 36 and $48 \mathrm{IU}$, it is possible that effects would have been detected at additional doses with larger samples. Third, it is possible that the OT effects are moderated by sex [23]. However, we did not have the power to examine for sex effects in the current design. Fourth, we did not have a healthy comparison group. Other OT studies have found effects at doses lower (e.g., $24 \mathrm{IU}$ ) than those in which we found effects in our study [23]. Having a healthy control group would have been informative regarding dose-responsiveness in people with schizophrenia. Finally, we did not collect information on nicotine use (either status or use prior to testing) which could have potentially impacted the results.

In conclusion, the results of the current study demonstrate target engagement of brain-based measures of social processing in people with schizophrenia at doses of 36 and 48 IU. These doses are within the range of those used in prior studies and lend confidence that these doses have a direct effect on the brain. Beyond providing guidance for specific doses, having a measure of target engagement will be useful for future studies of OT in schizophrenia. It may also have utility in demonstrating why some participants respond to OT while others may not. Finally, measures of target engagement may be a useful mediator that can be used to understand how to improve social processing in other psychiatric disorders that are characterized by difficulties in processing social information.

\section{ACKNOWLEDGEMENTS}

This material is the result of work supported with resources and the use of facilities at the University of California, Los Angeles Translational Research Center for Neuropsychiatry, the VA Greater Los Angeles Healthcare System (VAGLAHS) VISN 22 Mental IIIness Research, Education, and Clinical Center (SRM, Director), and the VAGLAHS Research Enhancement Award Program on Enhancing Community Integration for Homeless Veterans (MFG, Director). We thank Kumari Karunaratne and Jennifer Hoy for assistance with patient management and data collection. Funding support for this project was provided by NIMH grant 4R21MH107564 to S.R. $M$. The funding agency had no role in the design of the study, collection and analysis of data, or decision to publish. The contents do not represent the views of the U.S. Department of Veterans Affairs or the United States Government.

\section{ADDITIONAL INFORMATION}

Supplementary Information accompanies this paper at (https://doi.org/10.1038/ s41386-018-0165-y)

Competing interests: Dr. Marder has served as a consultant to Roche, Allergan, Takeda, Neurocrine, Newron, Boeringer-Ingelheim, Otsuka, Acadia, Lundbeck, and Teva. He has received research support from Neurocrine and Takeda. Dr. Green has been a consultant to AiCure, Lundbeck, and Takeda, and he is on the scientific board of Cadent. He has received research funds from FORUM. The remaining authors declare no competing interests.

Publisher's note: Springer Nature remains neutral with regard to jurisdictional claims in published maps and institutional affiliations.

\section{REFERENCES}

1. Lim MM, Young LJ. Neuropeptidergic regulation of affiliative behavior and social bonding in animals. Horm Behav. 2006:50:506-17.

2. Meyer-Lindenberg A, Domes G, Kirsch P, Heinrichs M. Oxytocin and vasopressin in the human brain: social neuropeptides for translational medicine. Nat Rev Neurosci. 2011;12:524-38.

3. Guastella AJ, Mitchell PB, Dadds MR. Oxytocin increases gaze to the eye region of human faces. Biol Psychiatry. 2008;63:3-5.

4. Bartholomeusz CF, Ganella EP, Labuschagne I, Bousman C, Pantelis C. Effects of oxytocin and genetic variants on brain and behaviour: Implications for treatment in schizophrenia. Schizophr Res. 2015;168:614-27.

5. Green JJ, Hollander E. Autism and oxytocin: new developments in translational approaches to therapeutics. Neurotherapeutics. 2010;7:250-7.

6. Insel TR, Gogtay N. National Institute of Mental Health clinical trials: new opportunities, new expectations. JAMA Psychiatry. 2014;71:745-6. 
7. Insel TR. Translating oxytocin neuroscience to the clinic: a National Institute of Mental Health Perspective. Biol Psychiatry. 2016;79:153-4.

8. Oberman LM, Ramachandran VS, Pineda JA. Modulation of Mu suppression in children with autism spectrum disorders in response to familiar or unfamiliar stimuli: the mirror neuron hypothesis. Neuropsychologia. 2008;46:1558-65.

9. Pineda JA. The functional significance of mu rhythms: translating "seeing" and "hearing" into "doing". Brain Res Rev. 2005;50:57-68.

10. Hobson HM, Bishop DVM. The interpretation of Mu suppression as an index of mirror neuron activity: past, present and future. R. Soc. Open Sci. 2017; 4:160662.

11. Perry A, Bentin S, Shalev I, Israel S, Uzefovsky F, Bar-On D, et al. Intranasal oxytocin modulated EEG mu/alpha and beta rhythms during perception of biological motion. Psychoneuroendocrinology. 2010;35:1446-53.

12. Prehn K, Heekeren HR, Blasek K, Lapschies K, Mews I, van der Meer E. Neuroticism influences pupillary responses during an emotional interference task. Int J Psychophysiol. 2008;70:40-9.

13. Siegle GJ, Granholm E, Ingram RE, Matt GE. Pupillary and reaction time measures of sustained processing of negative information in depression. Biol Psychiatry. 2001;49:624-36.

14. Prehn K, Kazzer P, Lischke A, Heinrichs M, Herpertz SC, Domes G. Effects of intranasal oxytocin on pupil dilation indicate increased salience of socioaffective stimuli. Psychophysiology. 2013;50:528-37.

15. Ventura J, Lukoff D, Nuechterlein KH, Liberman RL, Green MF, Shaner A. Brief psychiatric rating scale (BPRS). Int J Methods Psychiatr Res. 1993;3:227-43.

16. Kopelowicz A, Ventura J, Liberman RL, Mintz J. Consistency of brief psychiatric rating scale factor structure across a broad spectrum of schizophrenia patients. Psychopathology. 2008;41:77-84.

17. First MB, Spitzer RL, Gibbons M, Williams JBW. The Structured Clinical Interview for DSM-IV Axis I Disorders-Patient Edition. New York, NY: Biometrics Research; 1997.

18. Paloyelis Y, Doyle OM, Zelaya FO, Maltezos S, Williams SC, Fotopoulou A, et al. A spatiotemporal profile of in vivo cerebral blood flow changes following intranasal oxytocin in humans. Biol Psychiatry. 2016;79:693-705.
19. Born J, Lange T, Kern W, McGregor GP, Bickel U, Fehm HL. Sniffing neuropeptides: a transnasal approach to the human brain. Nat Neurosci. 2002;5:514-6.

20. Weisman O, Zagoory-Sharon O, Feldman R. Intranasal oxytocin administration is reflected in human saliva. Psychoneuroendocrinology. 2012;37:1582-6.

21. Matsumoto D, Ekman P. Japanese and Caucasian facial expressions of emotion (JACFEE) and neutral faces (JACNeuF). Dr. Paul Ekman, Department of Psychiatry, University of California, San Francisco. 1988.

22. Spengler FB, Schultz J, Scheele D, Essel M, Maier W, Heinrichs M, et al. Kinetics and dose dependency of intranasal oxytocin effects on amygdala reactivity. Biol Psychiatry. 2017;82:885-94.

23. Singh F, Nunag J, Muldoon G, Cadenhead KS, Pineda JA, Feifel D. Effects of intranasal oxytocin on neural processing within a socially relevant neural circuit. Eur Neuropsychopharmacol. 2016;26:626-30.

24. Marwick K, Hall J. Social cognition in schizophrenia: a review of face processing. Br Med Bull. 2008;88:43-58.

25. Statucka M, Walder DJ. Efficacy of social cognition remediation programs targeting facial affect recognition deficits in schizophrenia: a review and consideration of high-risk samples and sex differences. Psychiatry Res. 2013;206:125-39.

26. Wu EXW, Laeng B, Magnussen S. Through the eyes of the own-race bias: eyetracking and pupillometry during face recognition. Soc. Neurosci. 2012;7:202-16.

27. Grossman E, Donnelly M, Price R, Pickens D, Morgan V, Neighbor G, et al. Brain areas involved in perception of biological motion. J Cogn Neurosci. 2000;12:711-20.

28. Saygin AP, Wilson SM, Hagler DJ Jr., Bates E, Sereno MI. Point-light biological motion perception activates human premotor cortex. J Neurosci. 2004;24:6181-8.

29. Calabrese EJ, Baldwin LA. The frequency of $U$-shaped dose responses in the toxicological literature. Toxicol Sci. 2001;62:330-8. 